they are far in the heart of the gulf, away from any recent entanglement with the mainland, which is not quite so true of Prince Edward Island. Here is a cluster of rock fragments knit together by sand bars which show no single trace or semblance of recent elevation or depression. Even the broad dunecovered bars patched with stunted spruce and dune-grass afford no indication of tree burial or poisoning by encroachment of the water without or of the great lagoons within. The rocks of the islands are rather homogeneous in quality, except for the volcanics. The sandstones are retreating rapidly under the wave attacks, and while the volcanics stand out in better resistance, the broad submarine platform about the islands is uniformly smoothed. The soundings of the admiralty chart show how uniform the smoothing has been. The five-fathom platform ties all the islands of the Magdalens proper into one. The walrus bones heaped together on the top of the low horizontal rock shelves where they were left by the hunters more than a century ago, lie as they lay then, only coated with a century of soil and quietly falling away into the sea as the waters gnaw down the rocks. The five-fathom level is approximately a true wave platform, but the ten-fathom level, which outlines a platform of a hundred times the present superficies of the surviving islands, is unquestionably a wave-cut level deeply submerged. In this ten-fathom level there is no appeal from the evidence of a submergence at a time not far back of the present or from the conclusion that the Magdalens are mere interwoven shreds of a once great island, but we must not be pressed to declare how long ago the negative movement ceased. Not long, probably; but for this day, this present, we lack the right to say that there is any movement in process, up or down. A clue is suggested as to the length of this actual stability; facing the great interior lagoon bounded by the double chain of sand bars are ragged rock cliffs, with bare faces that never could have been torn by the feeble waters of the lagoon even in times of tempest. These cliffs, now enmeshed by sand and facing only placid waters, were made ragged and bare in the days when the sea itself pounded at their base. Since then the whole network of sand has been built up about them, and yet all this without any definite indication of change of water level.

\section{John M. Clarke}

\section{Einführung in die Mathematik für Biologen} und Chemiker. Von Professor Dr. Leonor Michaelis, Privatdozent an der Universität Berlin. Verlag von Julius Springer, Berlin. 1912.

It is the purpose of this book to bring before the chemist and the biologist; in convenient form, some mathematical information that is necessary to an understanding of methods that are being used more and more in chemistry and biology. The first chapter of the book gives a recapitulation of some very elementary mathematics, including plane geometry, the most elementary algebra and trigonometry. The second chapter is given to the study of some very simple functions such as are usually treated in a first course in analytic geometry. The main part of the book is given to the calculus, to differential equations, and to applications to chemistry and physics.

The author has succeeded in bringing a large amount of useful material into a small space, and the book will perhaps serve well its purpose. Although the reviewer recognizes that, in an elementary book, one may sacrifice too much simplicity for the sake of precision in the statement of fundamentals, there is some danger that the chemist and biologist will get incorrect views as to the precision of the processes of differentiation and integration when presented as they are in this book. To illustrate, on p. 107, we find the statement

$$
\sin \frac{d x}{2}=\frac{d x}{2}
$$

and analogous statements are to be found at many points in the book.

I note the following numerical and typographical errors: Line 23, p. 37 , should contain 0.7071 instead of 0.7069 , and line 9, p. 107, should have 
instead of

$$
\frac{\sin \pi / 2}{\pi / 2}=\frac{1}{1.57}
$$

$$
\frac{\sin \pi / 2}{\pi / 2}=\frac{0}{1.58}
$$

In carrying out his purposes, the author has very properly included a brief treatment of exact and inexact differentials, Fourier's series, and the application of imaginary numbers to the solution of some differential equations that are important in mathematical physics.

The final chapter of the book is devoted to directions for the representation of experimental data by mathematical functions, but the presentation is so brief that it is doubtful if the biologist or chemist could carry the directions into numerical effect without more mathematics than is given in this book.

On the whole, the author has shown good judgment in the selection of material for his purposes, and the biologist and chemist not familiar with the calculus will find the book of value.

\section{H. L. RiETZ}

\section{Radioactive Substances and Their Radiations.}

By E. Rutherford. Cambridge, University Press. 1913. Pp. vii +700 . Price, $\$ 4.50$.

The subject of radioactivity is now just sixteen years old, yet the volume of its literature already compares favorably with that of any of the other grand divisions of physics and two compendious text-books, Rutherford's and Madame Curie's-not to mention a host of less pretentious treatments-are available to initiate the student into its mysteries.

It is now eight years since the second edition of Rutherford's "Radioactivity" appeared, and in view of the fact that this period covers one half of the life of the science, it is scarcely to be expected that its present status could be adequately presented by a mere revision of that book. And it is to the author's credit that he has not attempted to patch the new material into the old frame, but has instead built an entirely new framework and merely utilized the old lumber wherever it still proved serviceable.
Out of a total of 700 pages, only about 150 are taken from the former work. Despite this fact, the present book makes very much the same impression as did its predecessor, whether it is given merely a cursory glance or whether it is made the subject of careful study. This is because the big problems of radioactivity were correctly solved at the start, and that largely by Rutherford himself. It is one of the most notable facts connected with this notable subject that within eight years of the discovery of the first radioactive rays, the phenomena of radioactivity should have been so thoroughly worked out and so unerringly interpreted that scarcely a viewpoint then taken in a book of 560 pages needs, after eight more years of exceedingly active experimenting, to be discarded.

The differences between the old book and the new are to be found not so much in method of treatment or in order of presentation, as in the incorporation of the new material which has accumulated within the past eight years. Much of this material has grown out of researches conducted in Rutherford's own laboratory. The additions have come chiefly from the careful study of the following subjects, none of which are found in the old text.

1. The range of the alpha particle, the law of its scattering in passing through matter, and the stopping power of substances for it. Through studies in these fields has come a great addition to our knowledge of the nature of the atom and the character of radioactive changes.

2. The phenomena of recoil, undiscovered when the old book was written, but recently diligently studied and shown to be invaluable as a means of separating radioactive products.

3. The methods of directly counting the alpha particles, one of which, namely, the scintillation method, has recently been of great help in the study of the short-lived products.

4. The scattering and change in velocity of the Beta rays in passing through matter and the remarkable resolution into a large number of homogeneous components of the Beta rays emitted by Radium $C$-studies which have thrown new light on the nature of the atom. 